\title{
CATALOGUE OF BIRDS COLLECTED BY SALIM ALI PRESENT IN THE NATURAL HISTORY MUSEUM, OSMANIA UNIVERSITY, HYDERABAD, INDIA
}

\section{Srinivasulu ${ }^{1}$, Bhargavi Srinivasulu and V. Nagulu}

Wildlife Biology Section, Department of Zoology, Osmania University, Hyderabad, Andhra Pradesh 570007, India Email: ${ }^{1}$ hyd2_masawa@sancharnet.in

\begin{abstract}
A catalogue of birds collected by Salim Ali during the Hyderabad State Ornithological Survey (1931-32) present in the collection of Natural History Museum of Osmania University, housed in Department of Zoology, Osmania University, Hyderabad, India is provided. This catalogue includes details on one quarter of the specimens collected during the Survey from 13 locations representing 191 specimens of 139 species and subspecies from 43 families.

KEYWORDS

Catalogue, Hyderabad State Ornithological Survey, Salim Ali, Natural History Museum, Osmania University.
\end{abstract}

Since its donation to the Natural History Museum of the Department of Zoology, Osmania University (henceforth, NHM.OU), as promised by Late. Dr. Salim A. Ali, to the then Nizam of the erstwhile Hyderabad State in return to the monetary support rendered to carry out the Hyderabad State Ornithological Survey (1931-32) - the first of its kind, the collection remained unknown for a period of almost seven decades. This collection represents about one quarter of the specimens collected during the Survey.

The first serious attempt to study the collection in detail and prepare a complete list of the same was carried out by us between 1995 and 1996 resulting in unpublished documentation present in the NHM.OU (Srinivasulu et al., 1996). The prepared list derives information basically from the tags attached to the specimens and the reports published on the Hyderabad State Ornithological Survey published in five parts in the Journal of the Bombay Natural History Society (Volumes 36 \& 37) in 1933 and 1934. Beside these reports and mention about the proceedings of the survey in Ali (1985), there exists no authentic written or published information on this collection.

Brief note on Hyderabad State Ornithological Survey Late Dr. Salim Ali started his monumental work on birds of the Indian subcontinent after having realized that the information regarding the bird life of the region is too scarce and undertook systematic bird surveys in the then 'Princely States' and selected regions of the then 'Provinces' under British regime. He kick started his studies after successful deliberations with the higher officials in Hyderabad State to permit and fund his project on documentation of bird diversity of the state. The project came to be known as the Hyderabad State Ornithological Survey that was carried out between October 1931 and April 1932.

The erstwhile Hyderabad State, encompassing a vast stretch (a polygonal tract covering an area of $2,14,187 \mathrm{~km}^{2}$ occupying the center of Deccan Plateau with an average altitude of c. $1250 \mathrm{ft}$ above sea level) of the now Telengana and Marthawada regions of Andhra Pradesh and Maharashtra states, was located between latitudes $15^{\circ} 10^{\prime}-20^{\circ} 40^{\prime} \mathrm{N} \& 74^{\circ} 40^{\prime}-81^{0} 35^{\prime} \mathrm{E}$. According to Ali (1933a) the Hyderabad State in some parts was mountainous, wooded and picturesque, in others flat and undulating with a mean temperature of $81^{\circ} \mathrm{F}$ and rainfall averaging between 30-32 inches annually.

The Hyderabad State Ornithological Survey, conducted in two phases, commenced collection at Mannanur in Mahbubnagar district on 3 October 1931 and continued until 20 December 1931 with last collections made at Asifabad in Adilabad district in the first phase. The works of the second phase commenced on last day of February 1932 at Kandahar in Nanded district and spanned till the end of April 1932 with last collections made at Bhamarvai in Nanded district. The Survey had to be curtailed due to lack of funding. Salim Ali also collected birds while at Hyderabad and also enroute to Mannanur.

Brief particulars of the localities from where the birds were collected during the Survey are provided below. For further details refer Ali (1933a).

Hyderabad City Environs (Hyderabad District): Survey period - 25 September to 3 October 1931. Altitude - 1,800ft (545.5m). Remarks - Broken slightly hilly country.

Mannanur (Mahbubnagar District): Survey period - 3 to 15 October 1931. Altitude - 2,000ft (606.1m). Remarks - On Amarabad Plateau. Amarabad Reserve Forest consists of mixed deciduous species as Tectona grandis and Terminalia tomentosa.

Farahabad (Mahbubnagar District): Survey period - 15 to 20 October 1931. Altitude - 2,800ft (848.5m). Remarks - 12 miles south of Mannanur on another plateau rising above Amarabad plateau. Country similar to Mannanur except on the whole more thickly wooded and containing a good deal more bamboo.

Borgampad (Warangal District): Survey period - 29 October to 4 November 1931. Altitude - 60ft (18.2m). Remarks - On the Godavari river opposite Bhadrachalam in the Madras Presidency (presently in Khammam district of Andhra Pradesh).

Nelipaka (Warangal District): Survey period - 18 November 1931. Altitude - 160ft (48.5m). Remarks - 9 miles from Borgampad, also on Godavari river. Scrub jungle giving place for the mixed deciduous forests.

Paloncha (Warangal District): Survey period - 18 to 26 
November 1931. Altitude - 300ft (90.9m). Remarks - 7 miles off Borgampad. Scrub and secondary jungle extremely thick making for poor visibility.

Narsampet (Warangal District): Survey period - 21 November to 9 December 1931. Altitude - $800 \mathrm{ft}(242.4 \mathrm{~m})$. Remarks - Locality dotted over with numerous tanks. In country open grassy patches among the scrub jungle. Collections were also made near Pakhal lake $\left(17^{0} 57^{\prime} \mathrm{N} \& 7^{0} 59^{\prime} \mathrm{E}\right)$, which is 6 miles east of Narsampet.

Asifabad (Asifabad District): Survey period - 10 to 20 December 1931. Altitude - 1250ft (378.8m). Remarks - Formerly known as Jangaon. Located in the Satmala Range. Scrub jungle on the hills which are 500-1000ft above the undulating plains. At the foothills of the Satmala range there is a good deal of bamboo and hillsides are covered with deciduous forests interspersed with patches of scrub, wood and are scourned everywhere with ravines and nullah on the sides of which much thin bamboo is present.

Kandahar (Nanded District): Survey period - 1 to 6 March 1932. Altitude - 1400ft (424.2m). Remarks - Stoney undulating country with long gently rolling hillocks; bare except for sparse stunted bushes mostly of a species of Randia and Gymnosporea montana.

Mukher (Nanded District): Survey period - 7 to 10 March 1932. Altitude - 1350ft (409.1m). Remarks - Located 18 miles southeast of Kandahar. Black cotton soil country undulating and cut up by lines of low rolling hillocks and shallow ravines.

Kaulas (Nanded District): Survey period - 17 to 28 March 1932. Altitude - 1300ft (393.9m). Remarks - rocky hills and ridges covered with deciduous jungle, bare in many places, with thin straggly teak here and there.

Utnoor (Asifabad District): Survey period - 1 to 10 April 1932. Altitude - 1250ft (378.8m). Remarks - Country similar to Asifabad mostly broken with nullahs and ridges on stony hills covered with scrub jungle. There is open deciduous and high grass jungle to the North, dense mixed jungle to the South and dense jungle, high grass and scattered bamboo among the hills to the East.

Kannad (Aurangabad District): Survey period - 15 to 18 April 1932. Altitude - 2000ft (606.1m). Remarks - 32 miles northwest of Aurangabad and 18 miles from the famous rock caves of Ellora. Hilly, broken country similar to that of Kaulas.

Bhamarvai (Aurangabad District): Survey period - 19 to 25 April 1932. Altitude - 2500ft (757.6m). Remarks - About 7 miles northwest of Kannad on the Outram Ghat on the boundary of Nizam's Dominion and Khandesh. The country is mostly hilly, broken, and undulating deciduous forest in which Boswellia serrata is very prominent.

\section{Catalogue}

We have arranged the collection according to the taxonomic classification of birds following Ali \& Ripley (1987). Scientific and common names follow Manakadan \& Pittie (2001) up to species level. Scientific nomen of subspecies is following Ripley (1982). For convenience, the collection was numbered according to the families. The first line for each entry in the present Catalogue includes Ripley's Synopsis number (see Ripley, 1982); current valid scientific name with the author name and year, and the common name. The second line includes the original names as given by Salim Ali (marked SA, if they differed from the currently accepted nomen) and other nomen (marked $\mathrm{O}$, nomen either valid to the subspecies in concern or alternative common nomen of the concerned taxon). The third line includes Natural History Museum registration number, original collection number in parenthesis, and details on sex of the specimen, date of collection and place of collection. Other details about the specimen provided by Salim Ali present on tags and also from his reports (Ali, 1933a, b, c; 1934a, b) are provided under the head 'Remarks'.

\section{Conclusion}

In total, about 750 specimens belonging to around 293 taxa (species and subspecies) were collected during the Hyderabad State Ornithological Survey that spanned for about five months conducted in two bouts (one each in 1931 and 1932). Of this vast collection, about 191 spezcimens belonging to 139 species belonging to 43 families are in the collection of the Natural History Museum of Department of Zoology, Osmania University, Hyderabad. We are unable to trace any documents detailing when and how many specimens did Dr. Salim Ali deposit or how the Collection found its way to the Department's Natural History Museum.

\section{REFERENCES}

Ali, S. (1933a). The Hyderabad State Ornithological Survey. Part 1. Journal of the Bombay Natural History Society 36(2): 356-390.

Ali, S. (1933b). The Hyderabad State Ornithological Survey. Part 2. Journal of the Bombay Natural History Society 36(3): 707-725.

Ali, S. (1933c). The Hyderabad State Ornithological Survey. Part 3. Journal of the Bombay Natural History Society 36(4): 898-919.

Ali, S. (1934a). The Hyderabad State Ornithological Survey. Part 4. Journal of the Bombay Natural History Society 37(1): 124-142.

Ali, S. (1934b). The Hyderabad State Ornithological Survey. Part 5. Journal of the Bombay Natural History Society 37(2): 425-454.

Ali, S. (1985). Fall of the Sparrow. Oxford University Press, Bombay, 265pp.

Ali, S. and S.D. Ripley (1987). Compact Handbook of the Birds of India and Pakistan. 2nd ed. Oxford University Press, Bombay.

Manakadan, R. and A. Pittie (2001). Standardised common and scientific names of the birds of the Indian subcontinent. Buceros 6(1): iix $+1-37$.

Ripley, S. D. (1982). A Synopsis of Birds of India and Pakistan. $2^{\text {nd }}$ ed. Oxford University Press- BNHS, Bombay.

\section{ACKNOWLEDGEMENTS}

We thank the Head, Department of Zoology, Osmania University for facilities. The first two authors acknowledge individual research grants by CSIR, New Delhi. 
Family: Anatidae

88 Dendrocygna javanica (Horsfield, 1821)

Lesser Whistling-Duck

SA: Lesser or Common Whistling Teal.

NHM.OU.HSOS.1. [407] Specimen of unknown sex 370 Vanellus malabaricus (Boddaert, 1783)

collected on 2.xii.31 from Narsampet (c' 820ft Yellow-wattled Lapwing

(248.5m)).

Remarks: Wing: 209mm.

114 Nettapus coromandelianus (Gmelin, 1789)

Cotton Teal

No nomen change.

NHM.OU.HSOS.la. [405] Specimen of unknown sex

collected on 2.xii.31 from Narsampet (c' $820 \mathrm{ft}$

(248.5m)).

Remarks: Wing: 167mm. Fresh plumage.

Family: Accipitiridae

157 Butastur teesa (Franklin, 1832)

White-eyed Buzzard

SA: White-eyed Buzzard-Eagle.

NHM.OU.HSOS.2. [99] Male collected on 11.x.31 from

Mannanur (c' 2,060ft (624.2m)).

Remarks: Testes undeveloped. Moulting on primaries.

190 Circus macrourus (S.G. Gmelin, 1770)

Pallid Harrier

SA: Pale Harrier.

NHM.OU.HSOS.2a. [356] Male collected on 23.xi.31 from Paloncha (c' 300ft $(90.9 \mathrm{~m})$ ).

Remarks: Testes developed.

Family: Falconidae

222 Falco tinnunculus tinnunculus (Linnaeus, 1758)

Common Kestre

SA: Cerchneis tinnunculus tinnunculus Linn. Kestrel. O: European Kestrel.

Female NHM.OU.HSOS.3. [215] Female collected on

1.xi.31 from Borgampad (c' 160ft). NHM.OU.HSOS.3a.

[318] Male collected on 15.xi.31 from Nelipaka (c'

$162 f t(49.1 \mathrm{~m})$ ).

Remarks: Female: Wing: $251 \mathrm{~mm}$. Ovaries undeveloped. Male: Wing: 237mm. Testes developed.

Family: Phasianidae

255 Perdicula asiatica asiatica (Latham, 1790)

Jungle Bush-Quail

No nomen change.

NHM.OU.HSOS.4. [310] Male collected on 14.xi.31 from Nelipaka (c' $200 \mathrm{ft}(60.6 \mathrm{~m}))$.

Remarks: Wing: $79 \mathrm{~mm}$. Testes developed; one bigger, $c^{\prime} 2 \times 7 \mathrm{~mm}$, than other. General plumage very worn, moulting on rump, flanks, etc.

Family: Rallidae

337 Porzana pusilla pusilla (Pallas, 1776)

Baillon's Crake

SA: Eastern Baillon's Crake.

NHM.OU.HSOS.5. [392] Immature female collected on 30.xi.31 from Narsampet (c' 780ft (236.4m)).

Remarks: Wing: $90 \mathrm{~mm}$. Ovaries undeveloped. Skull soft in center.

343 Amaurornis phoenicurus phoenicurus (Pennant, 1769)

White-breasted Waterhen

SA: Chinese White-breasted Water-hen

NHM.OU.HSOS.5a. [192] Male collected on 30.x.31 from Borgampad (c'160ft (48.5m)).

Remarks: Testes undeveloped. In general moult.

Family: Charadriidae

369 Vanellus duvaucelii (Lesson, 1826)

River Lapwing

SA: Hoplopterus duvaucelii (Lesson) Spur-winged

Plover. O: Spur-winged Lapwing.

NHM.OU.HSOS.6. [194] Male collected on 30.x.31

from Borgampad (c' 160ft (48.5m)).

SA: Lobipluvia malabarica Boddaert. from Asifabad (c' 1,200ft (363.6m)).

Remarks: Ovaries developed. Pair to No. 483.

373 Pluvialis fulva (Gmelin, 1789)

Pacific Golden-Plover O: Eastern Golden Plover.

from Borgampad (c' 160ft (48.5m)).

changing to winter plumage.

401 Actitis hypoleucos (Linnaeus, 1758)

Common Sandpiper

SA: Tringa hypoleucos Linn. plumage Common Snipe

Fantail Snipe. breast, back etc.

417 Calidris temminckii (Leisler, 1812)

Temminck's Stint

SA: Erolia temminckii (Leisler) from Borgampad (c'160ft (48.5m)).

Family: Rostratulidae

Greater Painted-Snipe

SA: Painted Snipe

from Narsampet (c' 800ft (242.4m)).

Moulting on back.

(Linnaeus, 1758)

Black-winged Stilt

No nomen change. Borgampad (c'160ft $(48.5 \mathrm{~m})$ ).

Remarks: Ovaries under developed.

Family: Burhinidae

437 Esacus recurvirostris (Cuvier, 1829)

Great Stone-Plover

SA: Great Stone Plover.

Nelipaka (c' 160ft $(48.5 \mathrm{~m}))$.

Remarks: Testes developed.

Family: Glareolidae

Indian Courser

No nomen change. from Borgampad (c' 160ft (48.5m)).

Remarks: Ovaries undeveloped.
. Moulting on head, neck, Small Pratincole

SA: Indian Small Pratincole or Swallow-Plover. O: Small Indian Pratincole.

NHM.OU.HSOS.I0a. [223] Female collected on 2.xi.31 from Borgampad (c'160ft $(48.5 \mathrm{~m})$ ).

NHM.OU.HSOS.6a. [484] Female collected on 18.xii.31

Remarks: Wing: $150 \mathrm{~mm}$. Ovaries under (developed)

Family: Pteroclididae

487 Pterocles exustus (Temminck, 1825)

Chestnut-bellied Sandgrouse

SA: Pterocles exustus erlangeri Neumann Common Indian Sandgrouse.

(G) Golden Plover. NHM OU.HSOS.11. [459] Female collected on 13.xii.31

from Asifabad (c' 1,200ft (363.6m)).

NHM.OU.HSOS.6b. [183] Male collected on 29.x.31 Remarks: Wing: 175mm. Ovaries developed.

Remarks: Wing: 160mm. Testes undeveloped. Juvenile 492 Pterocles indicus (Gmelin, 1789)

NHM.OU.HSOS.6c. [233] Male collected on 3.xi.31 from Borgampad (c' 200ft $(60.6 \mathrm{~m})$ ).

Remarks: Wing: 110mm. Testes undeveloped. Fresh

Painted Sandgrouse

No nomen change

NHM.OU.HSOS.11a. [275] Female collected on 9.xi.31 from Nelipaka (c' 200ft (60.6m)). NHM.OU.HSOS.11b.

[598] Male collected on 23.iii.32 from Kaulas (c' 1450ft $(439.4 \mathrm{~m}))$.

Remarks: Female - Wing: 168mm; ovaries undeveloped. Male - Wing: $180 \mathrm{~mm}$; testes undeveloped.

409 Gallinago gallinago gallinago (Linnaeus, 1758)

SA: Capella gallinago gallinago (Linn.) Common or

Family: Columbidae

504 Treron phoenicoptera chlorigaster (Blyth,

Yellow-legged Green-Pigeon

NHM.OU.HSOS.6d. [187] Male collected on 29.x.31 SA: Crocopus phoenicopetrus chlorigaster (Blyth) .

Remarks: Testes undeveloped. Moulting on throat, NHM.OU.HSOS.12. [320] Female collected on 15.xi.31

NHM.OU.HSOS.6e. [224] Female collected on 2.xi.31

Remarks: Wing: 99mm. Ovaries under developed.

429 Rostratula benghalensis benghalensis (Linnaeus, 1758)

NHM.OU.HSOS.7. [432] Female collected on 7.xii.31

Remarks: Wing: $142 \mathrm{~mm}$. Ovaries undeveloped.

Family: Recurvirostridae

430 Himantopus himantopus himantopus

NHM.OU.HSOS.8. [202] Female collected on $31 . \times 31$

NHM.OU.HSOS.9. [281] Male collected on 10.xi.31

440 Cursorius coromandelicus (Gmelin, 1789)

NHM.OU.HSOS.10. [259] Female collected on 6.xi.31 from Nelipaka (c' 162ft (49.1m)).

Remarks: Wing: $181 \mathrm{~mm}$. Ovaries undeveloped.

506 Ducula aenea sylvatica (Tickell, 1833)

Green Imperial-Pigeon

SA: Muscadivora aenea sylvatica (Tickell) Imperial Green Pigeon. O: North Indian Imperial Green Pigeon NHM.OU.HSOS.12a. [162] Male collected on 18.x.31 from Farahabad (c' 2,688ft $(814.5 \mathrm{~m}))$.

Remarks: Wing: $221 \mathrm{~mm}$. Testes developed.

517 Columba livia intermedia (Strickland, 1844) Blue-rock Pigeon

O: Indian Blue-rock Pigeon

NHM.OU.HSOS.12b. [412] Male collected from Narsampet (c' 820ft $(248.5 \mathrm{~m})$ ).

Remarks: Testes (right side measuring $22 \mathrm{~mm}$ ), breeding

537 Streptopelia chinensis suratensis (Gmelin, 1789)

Spotted Dove

SA: Indian Spotted Dove.

NHM.OU.HSOS.12c. [169] Male collected on 18.x.31

from Farahabad (c' 2,688ft $(814.5 \mathrm{~m})$ ).

Remarks: Testes undeveloped.

541 Streptopelia senegalensis (Linnaeus, 1766) Little Brown Dove

SA: Streptopelia senegalensis cambayensis (Gmelin). O: Indian Little Brown Dove

NHM.OU.HSOS.12d. [482] Female collected on 18.xii.31 from Asifabad (c' 1,200ft (363.6m)).

Remarks: Wing: 128mm. Ovaries undeveloped. Moulting primaries and upper wing coverts.

Family: Psittacidae

546 Psittacula eupatria eupatria (Linnaeus, 1766) Alexandrine Parakeet

SA: Large Indian Paraquet. O: Large Ceylonese Parakeet

NHM.OU.HSOS.13. [176] Male collected on 19.x.31

from Farahabad (c' 2,688ft $(814.5 \mathrm{~m})$ ).

Remarks: Wing: $214 \mathrm{~mm}$. Testes undeveloped. 
550 Psittacula krameri manillensis (Bechstein, 1800) Remarks: Wing: 345mm. Ovaries developed. Rose-ringed Parakeet

SA: Rose-ringed Paraquet

NHM.OU.HSOS.13a. [25] Male collected on 5.x.31 from Mannanur (c' 2,100ft (363.6m)).

Remarks: Testes undeveloped.

558 Psittacula cyanocephala cyanocephala (Linnaeus, 1766)

Plum-headed Parakeet

SA: Blossom-headed Paraquet. O: Blossom-headed Parakeet

NHM.OU.HSOS.13b. [626] Male collected on 1.iv.32 from Utnoor (c' 1,250ft (378.8m)).

Remarks: Wing: 140mm. Testes undeveloped.

Family: Cuculidae

573 Cuculus varius varius (Vahl, 1797)

Brainfever Bird

SA: Hierococcyx varius Vahl Common Hawk-Cuckoo.

$O$ : Indian Brain Fever Bird.

NHM.OU.HSOS.14. [282] Specimen of unknown sex collected on 10.xi.31 from Nelipaka (c' 160ft (48.5m)). Remarks: None.

\section{Cacomantis passerinus (Vahl, 1797)}

Indian Plaintive Cuckoo

SA: Cacomantis merulinus passerinus (Vahl).

NHM.OU.HSOS.14a. [90] Male collected on 11.x.31

from Mannanur (c' 2,060ft (624.2m)).

Remarks: Testes undeveloped.

590 Eudynamis scolopacea scolopacea (Linnaeus, 1758)

Asian Koel

SA: Eudynamis scolopaceus scolopaceus (Linn.) Koel. O: Indian Koel.

NHM.OU.HSOS.14b. [165] Female collected on 18.x.31 Farahabad (c' 2,688ft $(814.5 \mathrm{~m})$ ).

Remarks: Ovaries undeveloped.

595 Phaenicophaeus viridirostris (Jerdon, 1840) Small Green-billed Malkoha

SA: Rhopodytes viridirostris (Jerdon)

NHM.OU.HSOS.14c. [97] Male collected on 11.x.31 from Mannanur (c' 2,060ft (624.2m)).

Remarks: Testes undeveloped.

Family: Strigidae

627 Bubo bubo benghalensis (Franklin, 1831)

Eurasian Eagle-Owl

SA: Bubo bubo benghalensis (Franklin) Indian Great

Horned Owl

NHM.OU.HSOS.15. [184] Male colleted on 29.x.31 from Borgampad (c' 160ft $(48.5 \mathrm{~m})$ ).

Remarks: Wing: $365 \mathrm{~mm}$. Testes undeveloped. Moulting on neck.

636 Glaucidium radiatum radiatum (Tickell, 1833) Jungle Owlet

SA: Glaucidum radiatum radiatum (Tickell). O: Barred Jungle Owlet.

NHM.OU.HSOS.15a. [635] Specimen of unknown sex collected on 3.iv.32 from Utnoor (c'1,250ft (378.8m)). Remarks: Wing: $132 \mathrm{~mm}$. (damaged). One of a pair in forest.

652 Athene brama brama (Temminck, 1821) Spotted Owlet

O: Southern Spotted Owlet

NHM.OU.HSOS.15b. [89] Female collected on 11.x.31 from Mannanur (c' 2,060ft (624.2m)).

Remarks: Ovaries undeveloped. Presumably first year

657 Strix ocellata ocellata (Lesson, 1839)

Mottled Wood Owl

SA: Mottled Wood-Owl. O: Southern Mottled Wood Owl

NHM.OU.HSOS.15c. [415] Adult female collected on

3.xii.31 from Narsampet (c' $800 \mathrm{ft}(242.4 \mathrm{~m})$ ).
Family: Caprimulgidae

680 Caprimulgus asiaticus asiaticus (Latham, 1790) Common Indian Nightjar

No nomen charges

NHM.OU.HSOS.16. [102] Male collected on 12.x.31

from Mannanur (c' 2,060ft (624.2m))

Remarks: Wing: $146 \mathrm{~mm}$. Testes developed. Genera moult, complete moult. Ali (1934a) mentions it as breeding male.

682 Caprimulgus affinis monticolus (Franklin 1831)

Franklin's Nightjar

SA: Caprimulgus monticolus monticolus Franklin.

NHM.OU.HSOS.16a. [324] Female collected on

Remarks: Ovaries undeveloped.

Family: Apodidae

707 Cypsiurus parvus balasiensis (J.E. Gray, 1829)

Asian Palm-Swift

SA: Palm Swift

NHM.OU.HSOS.17. [682] Male collected on 9.iv.32 from Utnoor (c' 1,250ft (378.8m)).

Remarks: Wings: $115 \mathrm{~mm}$. Testes developed, pulpy In general moult, body, wings and tail.

709 Hemiprocne coronata (Tickell, 1833)

Crested Tree-Swift

SA: Indian Crested Swift. O: Crested Swift.

NHM.OU.HSOS.17a. [637] Male collected on 3.iv.32

from Utnoor (c' 1,250ft (378.8m)).

Remarks: Testes developed, breeding. In body moult.

Incubation patch present.

Family: Meropodidae

744 Merops leschnaulti leschnaulti (Vieillot, 1817)

Chestnut-headed Bee-eater

SA: Melittophagus erythrocephalus erythrocephalus

(Gmelin) Chestnut-headed Bee-eater

NHM.OU.HSOS.18. [277] Female collected on 9.xi.31 from Nelipaka (c' 200ft (60.6m)).

Remarks: Wing: 109mm. Culmen: $37.5 \mathrm{~mm}$. Ovaries undeveloped.

Family: Coraciidae

756 Coracias benghalensis indica (Linnaeus, 1766) Indian Roller

No nomen change

NHM.OU.HSOS.19. [368] Male collected on 24.xi.31 from Paloncha (c' 300ft(90.9m)).

Remarks: Wing: 182mm. Testes undeveloped. Fresh plumage.

Family: Upupidae

765 Upupa epops ceylonensis (Reichenbach, 1853)

Common Hoopoe

SA: Upupa epops ceylonensis Reichenbach Ceylon

Hoopoe. O: Indian Hoopoe.

NHM.OU.HSOS.20. [350] Male collected on 21.xi.31

from Paloncha (c' 300ft $(90.9 \mathrm{~m})$ ).

Remarks: Testes-only left testis present. Generally in

fresh plumage.

Family: Capitonidae

782 Megalaima zeylanica zeylanica (Gmelin, 1788)

Brown-headed Barbet

SA: Thereiceryx zeylonicus (Gmelin) Green Barbet. O Large Green Barbet.

NHM.OU.HSOS.21. [27] Male collected on 5.x.31 from Mannanur (c'2,100ft (636.4m)).

Remarks: Testes undeveloped.

792 Megalaima haemacephala indica (Latham, 1788)

Coppersmith Barbet

SA: Xantholaema haemacephala indica (Latham) 16.xi.31 from Nelipaka (c' 162ft (49.1m)).
Crimson-breasted Barbet

NHM.OU.HSOS.21a. [81] Male collected on 10.x.31

from Mannanur (c'2,060ft (624.2m)).

Remarks: Testes undeveloped.

Family: Picidae

796 Jynx torquilla torquilla (Linnaeus, 1758)

Eurasian Wryneck

SA: Wryneck

NHM.OU.HSOS.22. [237] Female collected on 3.xi.31 from Borgampad (c' 200ft $(60.6 \mathrm{~m})$ ).

Remarks: Wing: $91 \mathrm{~mm}$. Ovaries undeveloped. Fresh plumage.

819 Dinopium benghalense benghalense (Linnaeus, 1758)

esser Golden-backed Woodpecker

SA: Brachypternus benghalensis Linn. Golden-backed Woodpecker.

NHM.OU.HSOS.22a. [98] Male collected on 11.x.31 from Mannanur (c' 2,060ft (624.2m)).

NHM.OU.HSOS.22b [211] Specimen of unknown sex collected on 1.xi.31 from Borgampad (c' 160ft (48.5m)). Remarks: Male: Moulting primaries and retrices.

Unknown sex: ?Moulting on head.

\section{Dendrocopus mahrattensis (Latham, 1801)}

Yellow-fronted Pied Woodpecker

SA: Dryobates mahrattensis mahrattensis (Latham)

$\mathrm{O}$ : Picoides mahrattensis mahrattensis (Latham)

Yellowfronted Pied Woodpecker.

NHM.OU.HSOS.22c. [393] Male collected on 30.xi.31

from Narsampet (c' 780ft $(236.4 \mathrm{~m})$ ).

NHM.OU.HSOS.22d. [629] Male collected on 2.iv.32

from Utnoor (c' 1,250ft $(378.8 \mathrm{~m})$ ).

Remarks: Male: Wing: $96.5 \mathrm{~mm}$; testes undeveloped; fresh plumage. Male: Wings: $103 \mathrm{~mm}$; testes developed, pulpy; incubation patch present.

852 Dendrocopus nanus hardwickii (Jerdon, 1844) Brown-capped Pygmy Woodpecker

SA: Dryobates hardwickii hardwickii (Jerdon) Pigmy

Woodpecker. O: Picoides nanus hardwickii (Jerdon).

NHM.OU.HSOS.22c. [59] Specimen of unknown sex

collected on 8.x.31 from Mannanur (c'2,060ft

$(624.2 \mathrm{~m}))$.

NHM.OU.HSOS.22f. [649] Male collected on 4.iv.32 from Utnoor (c' 1,250ft (378.4m)).

Remarks: Unknown Sex: Wing: 79mm. Male: Wing: $80 \mathrm{~mm}$; testes undeveloped.

Family: Pittidae

867 Pitta brachyura (Linnaeus, 1766)

Indian Pitta

SA: Pitta

NHM.OU.HSOS.23. [35] Male collected on 6.x.31 from Mannanur (c' 2,100ft (636.4m))

Remarks: Testes undeveloped.

\section{Family: Alaudidae}

874 Mirafra ajfinis (Blyth, 1845)

Jerdon's Bush-Lark

SA: Madras Bush-Lark. O: Mirafra assamica affinis

Blyth

NHM.OU.HSOS.24. [11] Male collected on 27.ix.31 near Umda Sagar (c'1,800ft $(545.5 \mathrm{~m})$ ) in Hyderabad City Environs.

NHM.OU.HSOS.24a. [12] Male collected on 27.ix.31 near Umda Sagar (c' 1,800ft $(545.5 \mathrm{~m}))$ in Hyderabad City Environs.

Remarks: Male - 1: Wing: 83mm; testes developed iris hazel brown; bill flesh, darker on culmen; feet and claws flesh, darker. Male - 2: Wing: $83 \mathrm{~mm}$; testes developed; iris hazel brown; bill culmen brown commissure and lower margin flesh; feet and claws flesh, darker

877 Mirafra erythroptera erythroptera (Blyth, 1845) Red-winged Bush-Lark 
No nomen change

NHM.OU.HSOS.24b. [13] Male collected on 27.ix.31 near Umda Sagar (c' 1,800ft $(545.5 \mathrm{~m})$ ) in Hyderabad City Environs.

NHM.OU.HSOS.24c. [14] Male collected on 27.ix.31 near Umda Sagar (c' 1,800ft (545.5m)) in Hyderabad City Environs.

Remarks: Male -1: Wing: 80mm; testes undeveloped; skull soft; iris hazel brown; bill flesh, darker on culmen; feet and claws flesh colour. Male - 2: Wing: $79 \mathrm{~mm}$; testes undeveloped; skull soft; iris hazel brown; bill flesh, darker on culmen; feet and claws flesh colour.

878 Ermeopterix grisea (Scopoli, 1786)

Ashy-crowned Sparrow-Lark

SA: Ashy-crowned Finch-Lark

NHM.OU.HSOS.24d. [65] Male collected on 8.x.31 from Mannanur (c' 2,060ft (624.2m)).

NHM.OU.HSOS.24e. [383] Female collected on 27.xi.31 from Paloncha (c' 300ft $(90.9 \mathrm{~m})$ ).

Remarks: Male: Testes developed. Female: Wing: $74 \mathrm{~mm}$; ovaries developed.

882 Ammomanes phoenicurus phoenicurus (Franklin, 1831)

Rufous-tailed Finch-Lark

No nomen change.

NHM.OU.HSOS.24f. [270] Male collected on 9.xi.31 from Nelipaka (c' 160ft (48.5m)).

NHM.OU.HSOS.24g. [462] Female collected on 13.xii.31 from Asifabad (c' 1,200ft (363.6m)).

Remarks: Male: Testes undeveloped. Female: Wing: $100.5 \mathrm{~mm}$; ovaries undeveloped.

902 Galerida deva (Sykes, 1832)

Sykes's Crested Lark

O: Deccan Crested Lark.

NHM.OU.HSOS.24h. [8] Female collected on 25.ix.31 near Umda Sagar (c' $1,800 \mathrm{ft}(545.5 \mathrm{~m}))$ in Hyderabad City Environs.

NHM.OU.HSOS.24i. [503] Immature male collected on 3.iii.32 from Kandahar (c'1,400ft (424.2m)).

Remarks: Female: Wing: $85 \mathrm{~mm}$; ovaries developed; soft eggs; iris hazel brown; bill horny-flesh, darker on under margin; feet and claws flesh colour. Immature male: Wing: $85 \mathrm{~mm}$; testes undeveloped; skull soft; very worn plumage, moulting last 2 secondaries.

Family: Hirundinidae

917 Hirundo rustica gutturalis Scopoli, 1786 Common Swallow

O: Eastern Swallow.

NHM.OU.HSOS.25. [64] Female collected on 8.x.31 from Mannanur (c' 2,060ft (624.2m)).

Remarks: Ovaries undeveloped.

Family: Laniidae

933 Lanius meridionalis (Temminck, 1820)

Southern Grey Shrike

SA: Lanius excubitor lahtora (Sykes, 1832). O: Indian Grey Shrike.

NHM.OU.HSOS.26. [23] Male collected on 5.x.31 from Mannanur (c' 2,100ft (636.4m)).

Remarks: Testes undeveloped. Wing and tail moult.

940 Lanius vittatus vittatus (Valencciennes, 1826) Bay-backed Shrike

SA: Baybacked Shrike. O: Indian Bay-backed Shrike. NHM.OU.HSOS.26a. [3] Male collected on 25.ix.31 near Umda Sagar (c' 1,800ft (545.5m)) near Hyderabad City Environs.

NHM.OU.HSOS.26b. [79] Female collected on 10.x.31 from Mannanur (c' 2,060ft $(624.4 \mathrm{~m})$ ).

Remarks: Male: Wing: $90 \mathrm{~mm}$; testes undeveloped; iris brown; bill horny brown; feet and claws plumbaceous. Female: Ovaries undeveloped; in heavy moult on neck, forehead and wings.
947 Lanius schach caniceps (Blyth, 1846)

Rufous-backed Shrike

A: Greybacked Shrike. O: South Indian Grey-backed Shrike.

NHM.OU.HSOS.26c. [230] Male collected on 3.xi.31 Borgampad (c' 200ft $(60.6 \mathrm{~m}))$.

Remarks: Wing: $86 \mathrm{~mm}$. Testes undeveloped. Genera moult, bases of flesh feathers ensheathed.

Family: Oriolidae

953 Oriolus oriolus kundoo (Sykes, 1832)

Eurasian Golden Oriole

SA: Golden Oriole.

NHM.OU.HSOS.27. [304] Female collected on 14.xi.31 from Nelipaka (c' 162ft (49.1m)).

Remarks: Wing: $141 \mathrm{~mm}$. Ovaries undeveloped.

Family: Dicruridae

963 Dicrurus macrocercus (Vieillot, 1817)

Black Drongo

SA: Dicrurus macrocercus peninsularis Ticehurst. NHM.OU.HSOS.28. [226] Immature female collected on 2.xi.31 from Borgampad (c' 160ft (48.5m)).

NHM.OU.HSOS.28a. [430] Male collected on 6.xii.31 from Narsampet (c' $800 \mathrm{ft}(242.4 \mathrm{~m})$ ) near Pakhal Lake. Remarks: Immature female: Wing: $136 \mathrm{~mm}$; ovaries undeveloped; skull soft. Male: Wing: $151 \mathrm{~mm}$; testes undeveloped; moult on throat, back; general plumage fresh.

967 Dicrurus caerulescens caerulescens (Linnaeus, 1758)

\section{White-bellied Drongo}

O: Indian White-bellied Drongo

NHM.OU.HSOS.28b. [49] Male collected on 7.x.31 from Mannanur (c'2,100ft (636.4m))

NHM.OU.HSOS.28c. [149] Male collected on 17.x.31 from Farahabad (c' 2,872ft $(870.3 \mathrm{~m})$ ).

NHM.OU.HSOS.28e. [173] Female collected on 9.x31 from Farahabad (c' 2,688ft (814.5m)).

Remarks: Male -1: Testes undeveloped; moulting secondaries. Male -2: Wing: $126 \mathrm{~mm}$; testes ndeveloped. Female: Wing: $123 \mathrm{~mm}$; ovaries undeveloped.

976 Dicrurus paradiseus grandis (Gould, 1836) Greater Racket-tailed Drongo

SA: Dissemurus paradiseus grandis (Gould.) Rackettailed Drongo. O: Northern Large Racket-tailed Drongo NHM.OU.HSOS.28d. [370] Male collected on 25.xi.3 from Paloncha (c' 500ft $(151.5 \mathrm{~m})$ ).

Remarks: Wing: $166 \mathrm{~mm}$. Tail: $393 \mathrm{~mm}$. Testes developed, very fat.

Family: Artamidae

982 Artamus fuscus (Vieillot, 1817)

Ashy Woodswallow

SA: Ashy Swallow-Shrike. O: Ashy Swallow Shrike. NHM.OU.HSOS.29. [179] Male collected on 19.x.3 from Farahabad (c' 2,793ft (837.3m)).

Remarks: Testes undeveloped.

Family: Sturnidae

987 Sturnus malabaricus malabaricus (Gmelin, 1789)

Grey-headed Starling

SA: Sturnia malabarica malabarica (Gmelin) Grey headed Myna

NHM.OU.HSOS.30. [447] Female collected on 8.xii.31 from Narsampet (c' 750ft (227.3m)).

Remarks: Wing: $101 \mathrm{~mm}$. Ovaries undeveloped. Fresh plumage.

994 Sturnus pagodarum (Gmelin, 1789)

Brahminy Starling

SA: Temenuchus pagodarum Black-headed Myna NHM.OU.HSOS.30a. [46] Male collected on 7.x.31 from Mannanur (c' 2,060ft (624.2m))

Remarks: Testes undeveloped.
996 Sturnus roseus (Linnaeus, 1758)

Rosy Starling

SA: Pastor roseus (Linn.) Rose-coloured Starling. O: Rosy Pastor.

NHM.OU.HSOS 30b. [373] Immature female collected on 25.xi.31 from Paloncha (c'300ft $(90.9 \mathrm{~m})$ ).

Remarks: Wing: $126 \mathrm{~mm}$. Ovaries undeveloped. Skull soft in center.

1002 Sturnus contra contra (Linnaeus, 1758)

Asian Pied Starling

SA: Sturnopastor contra contra (Linn.) Indian Pied Myna.

NHM.OU.HSOS.30c. [188] Male collected on 29.x.31 from Borgampad (c' 160ft (48.5m)).

Remarks: Testes undeveloped. Moulting primaries, retrices, and on throat, neck.

Family: Corvidae

1034 Dendrocitta vagabunda vernayi (Whistler \& Kinnear, 1930)

Indian Treepie

SA: South Indian Treepie. O: Southeastern Treepie

NHM.OU.HSOS.31. [26] Immature male collected on 5.x.31 from Mannanur (c' 2,100ft $(636.4 \mathrm{~m})$ ).

NHM.OU.HSOS.31a. [290] Male collected on 11.xi.31 Nelipaka (c' 160ft $(48.5 \mathrm{~m})$ ).

NHM.OU.HSOS.31b. [364] Adult female collected on 24.xi.31from Paloncha (c' 400ft (121.2m)).

Remarks: Immature male: Testes undeveloped; skull soft. Male: Wing: 155mm, testes undeveloped, moulting secondaries and on abdomen. Female: Wing $147 \mathrm{~mm}$; ovaries undeveloped; fresh plumage.

1049 Corvus splendens splendens (Vieillot, 1817) House Crow

AA: Indian House-Crow. O: Indian House Crow. NHM.OU.HSOS.31c. [204] Male collected on 31.x.31 from Borgampad (c'160ft $(48.5 \mathrm{~m})$ ).

Remarks: Testes undeveloped. Moulting primaries and on throat, neck.

1057 Corvus macrorhynchos culminatus (Sykes, 1832)

Jungle Crow

SA: Southern Jungle-Crow. O: Indian Jungle Crow. NHM.OU.HSOS.31d. [376] Male collected on 26.xi.31 from Paloncha (c'300ft (90.9m)).

NHM.OU.HSOS.31e. [465] Female collected on 13.xii.31 from Asifabad (c' 1,200ft (363.6m)).

Remarks: Male: Wing: $310 \mathrm{~mm}$; testes undeveloped

fresh plumage. Female: Ovaries undeveloped.

Family: Campephagidae

1070 Tephrodornis pondicerianus pondicerianus

(Gmelin, 1789)

Common Woodshrike

SA: Wood Shrike. O: Indian Wood Shrike.

NHM.OU.HSOS.32. [76] Male collected on 10.x.31 from Mannanur (c' 2,060ft (624.2m)).

NHM.OU.HSOS.32a. [431] Female collected on 6.xii.31 from Pakhal Lake (c' $800 \mathrm{ft}(242.4 \mathrm{~m})$ ).

Remarks: Male: Testes undeveloped. Female: Wing

$87 \mathrm{~mm}$, ovaries undeveloped.

1072 Coracina macei (Lesson, 1830)

Large Cuckoo-Shrike

SA: Graucalus javensis macei (Lesson) Large Indian A Goo-shrike.

NHM.OU.HSOS.32b. [163] Adult male collected on 18.x.31 from Farahabad (c' 2,688ft (814.5m)).

Remarks: Wing: $164 \mathrm{~mm}$. Testes undeveloped.

1079 Coracina melanoptera sykesi (Strickland, 1844)

Black-headed Cuckoo-Shrike

SA: Lalage sykesi (Strickland). O: Peninsular Blackheaded Cuckoo-Shrike.

NHM.OU.HSOS.32c. [20] Adult female collected on 
27.ix.31 near Umda Sagar (c' 1,800ft $(545.5 \mathrm{~m})$ in Family: Muscicapidae Hyderabad City Environs.

1154 Pellorneum ruficeps (Swainson, 1832)

Remarks: Wing: $105 \mathrm{~mm}$. Ovaries undeveloped. Iris Spotted Babbler

dark brown. Bill horny brown. Feet and claws horny No nomenchange brown.

1091 Pericrocotus cinnamomeus perigrinus (Linnaeus, 1766)

Small Minivet

SA: Pericrocotus perigrinus perigrinus (Linn.). O: Northern Small Minivet

NHM.OU.HSOS.32d. [115] Male collected on 13.x.31

from Mannanur (c' 2,100ft $(636.4 \mathrm{~m})$ ).

NHM.OU.HSOS.32e. [243] Immature specimen of unknown sex collected on 4.xi.31 from Borgampad ( $\mathrm{C}^{\prime}$ $200 \mathrm{ft}(60.6 \mathrm{~m}))$.

Remarks: Male: Wing: $64 \mathrm{~mm}$; testes undeveloped; entire moult just finishing. Unknown sex: $68 \mathrm{~mm}$; ?moulting on throat, neck, breast, back; skull soft.

\section{Pericrocotus erythropygius erythropygius} (Jerdon, 1840)

White-bellied Minivet

No nomen change

NHM.OU.HSOS.32f. [110] Male collected on 12.x.31 from Mannanur (c' 2,060ft $(624.2 \mathrm{~m})$ ).

Remarks: Wing: $66 \mathrm{~mm}$. Testes undeveloped. Moult on wings, head.

\section{Family: Irenidae}

1099 Aegithinia tiphia humei (Stuart Baker, 1922) Indian lora

SA: Central Indian lora.

NHM.OU.HSOS.33. [240] Immature specimen of unknown sex collected on 4.xi.31 from Borgampad (c' $200 f t(60.6 \mathrm{~m}))$.

NHM.OU.HSOS.33a. [257] Female collected on 6.xi.3 from Borgampad (c' 160ft (48.5m)).

Remarks: Unknown sex: ? In heavy body moult; Retrices fresh; skull soft. Female: Ovaries undeveloped; in heavy general moult.

1107 Chloropsis cochinchinensis jerdoni (Blyth, 1844)

\section{Jerdon's Chloropsis}

SA: Chloropsis jerdoni (Blyth).

NHM.OU.HSOS.33b. [60] Male collected on 8.x.31 from Mannanur (c' 2,060ft (624.2m)).

Remarks: Testes undeveloped.

\section{Family: Pycnonotidae}

1121 Pycnonotus jocosus emeria (Linnaeus, 1758)

Red-whiskered Bulbul

SA: Otocompsa jocose emeria (Linn.) Redwhiskered Bulbul. O: Bengal Red-whiskered Bulbul.

NHM.OU.HSOS.34. [242] Male collected on 4.xi.31 from Borgampad (c' 200ft $(60.6 \mathrm{~m})$ ).

Remarks: Wing: $83 \mathrm{~mm}$. Testes undeveloped. In heavy general moult.

1128 Pycnonotus cafer cafer (Linnaeus, 1766)

Red-vented Bulbul

SA: Molpastes cafer cafer.

NHM.OU.HSOS.34a. [186] Male (doubtful) collected on 29.x.31 from Borgampad (c' 160ft (48.5m)).

NHM.OU.HSOS.34b. [456] Female collected on 12.xii.31 from Asifabad (c' 1,200ft (363.6m)).

Remarks: Male: ?Moulting primaries, retrices and on abdomen. Female: Wing: $88 \mathrm{~mm}$; ovaries undeveloped; moulting primaries, retrices, head and throat.

1138 Pycnonotus luteolus luteolus (Lesson, 1841) White-browed Bulbul

No nomen change

NHM.OU.HSOS.34c. [289] Male collected on 11.xi.31 from Nelipaka (c' 160ft (48.5m)).

Remarks: Wing: $87 \mathrm{~mm}$. Testes (undeveloped). Ali

(1933a) writes that it is an "Adult in a quiescent state (of organs)". from Nelipaka (c' 160ft $(48.5 \mathrm{~m}))$ general moult.

1832)

Indian Scimitar Babbler from Farahabad (c' 2,872ft $(870.3 \mathrm{~m}))$.

Remarks: Wing: 99mm. Testes developed. 1831)

\section{Rufous-bellied Babbler}

No nomen change. from Borgampad (c' 200ft $(60.6 \mathrm{~m})$ ). $162 \mathrm{ft}(49.1 \mathrm{~m}))$ $162 \mathrm{ft}(49.1 \mathrm{~m}))$. from Narsampet (c' 800ft $(242.4 \mathrm{~m})$ ). (Ali, 1933a). Yellow-eyed Babbler SA: Chrysomma sinensis sinensis (Gmelin) $2,060 \mathrm{ft}(624.2 \mathrm{~m}))$. from Mannanur (c' 2,060ft (624.2m)). from Narsampet (c' 800ft $(242.4 \mathrm{~m})$ ). moult nearing completion. Common Babbler

SA: Argya caudata caudata (Dumont) from Asifabad (c' 1,200ft (363.6m)) City Environs brown; feet yellow, claws like bill.

1258 Turdoides malcolmi (Sykes, 1832) Large Grey Babbler from Kannad (c' 2,050ft $(621.2 \mathrm{~m})$ ).

Remarks: Ovaries undeveloped.
1264 Turdoides striatus malabaricus (Jerdon, 1847) Jungle Babbler

SA: Turdoides somervillei malabaricus (Jerdon) Southern Jungle-Babbler. O: Malabar Jungle Babbler

NHM.OU.HSOS.35. [319] Male collected on 15.xi.31 NHM.OU.HSOS.35m. [24] Female collected on 5.x.31

from Mannanur (c' 2,100ft $(636.4 \mathrm{~m}))$

Remarks: Wing: 73mm. Testes undeveloped. In heavy NHM.OU.HSOS.35n. [41] Male collected on 6.x.31

from Mannanur (c' 2,060ft $(624.4 \mathrm{~m})$ ).

Remarks: Female: Ovaries undeveloped. Male: Testes developed; breeding.

SA: Pomatorhinus horsfieldii Sykes Deccan Scimitar

NHM.OU.HSOS.35a. [148] Male collected on 17.x.31

1222 Dumetia hyperythra hyperythra (Franklin,

NHM.OU.HSOS.35b. [255] Female collected on 5.xi.31

NHM.OU.HSOS.35c. [315] Immature specimen of unknown sex collected on 15.xi.31 from Nelipaka (c

NHM.OU.HSOS.35d. [317] Immature specimen of unknown sex collected on 15.xi.31 from Nelipaka (c'

NHM.OU.HSOS.35e. [403] Male collected on 1.xii.31

Remarks: Female: Wing: $49 \mathrm{~mm}$; ovaries undeveloped in general moult. Unknown sex -1: Wing: $52 \mathrm{~mm}$; ?in general moult; skull soft in center. Unknown sex - 2 : ?In general moult; skull soft. Male: Testes (c' $3 x$ $2 \mathrm{~mm}$ ), pulpy in texture suggesting finished breeding

1231 Chrysoma sinense sinense (Gmelin, 1789)

NHM.OU.HSOS.35f. [94] Immature specimen of unknown sex collected on 11.x.31 from Mannanur (c

NHM.OU.HSOS.35g. [125] Male collected on 14.x.31

NHM.OU.HSOS.35h. [413] Male collected on 3.xii.31

NHM.OU.HSOS.35i. [469] Immature female collected on 15.xii.31 from Asifabad (c' 1,200ft (363.6m)).

Remarks: Unknown sex: None. Male: Wing: $68 \mathrm{~mm}$ testes developed; breeding. Male -2: Wing: $67 \mathrm{~mm}$; testes undeveloped; in heavy general moult including primaries, retrices; incubation patch on abdomen. Immature female: Wing: $63 \mathrm{~mm}$; ovaries undeveloped skull very soft; moulting primaries and retrices; body

1254 Turdoides caudatus caudatus (Dumont, 1823)

NHM.OU.HSOS.35j. [476] Male collected on 16.xii.31 NHM.OU.HSOS.35k. [2] Female collected on 25.ix.31 Mir Alam Tank (c' 1,800ft (545.5m)) Hyderabad

Remarks: Male: Testes undeveloped. Female: Wing $72 \mathrm{~mm}$; ovaries undeveloped; iris brown; bill fleshy SA: Argya malcolmi (Sykes).

NHM.OU.HSOS.35I. [705] Female collected on 7.iv.32
1267 Turdoides qffinis affinis (Jerdon, 1847)

White-headed Babbler

SA: Turdoides striatus polioplocamus Oberholser Whiteheaded Babbler.

NHM.OU.HSOS.35o. [48] Male collected on 7.x.31 from Mannanur (c' 2,060ft (624.2m))

NHM.OU.HSOS.35p. [292] Female collected on 11.xi.31 from Nelipaka (c' 160ft $(48.5 \mathrm{~m})$ ).

Remarks: Male: Testes developed; moulting on breast. Female: Wing: $107 \mathrm{~mm}$; ovaries undeveloped; in heavy general moult.

\section{Muscicapa dauurica (Pallas, 1811)}

Asian Brown Flycatcher

SA: Alseonax latirostris (Raffles) Indian Brown Flycatcher. O: Muscicapa latirostris Raffles Brown Flycatcher.

NHM.OU.HSOS.35q. [55] Specimen of unknown sex collected on 8.x.31 from Mannanur (c' 2,060ft (624.2m)).

Remarks: Wing: $70.5 \mathrm{~mm}$

1408 Muscicapa muttui muttui (Layard, 1854)

Brown-breasted Flycatcher

SA: Alseonax muttui muttui (Layard) Layard's Flycatcher.

NHM.OU.HSOS.35r. [130] Specimen of unknown sex $(636.4 \mathrm{~m}))$.

Remarks: Wing: $72 \mathrm{~mm}$. In fresh plumage.

1412 Ficedula parva albicilla (Pallas, 1811)

Red-throated Flycatcher

SA: Siphia parva albicilla (Pallas) Red-breasted Flycatcher. O: Muscicapa parva albicilla Pallas Eastern Red-breasted Flycatcher.

NHM.OU.HSOS.35s. [526] Female (doubtful) collected on 8.iii.32 Mukher (c' 1350ft (409.1m)).

Remarks: Wing: $67 \mathrm{~mm}$. Testes (? light). Moult on throat and below ear coverts. Very fat.

1421 Ficedula supercilliaris supercilliaris (Jerdon, 1840)

Ultramarine Flycatcher

SA: Muscicapula supercilliaris supercilliaris (Jerdon) White-browed Blue Flycatcher. O: Muscicapa supercilliaris supercilliaris Jerdon.

NHM.OU.HSOS.35t. [422] Female (doubtful) collected on 5.xii.31 Narsampet (c' 800ft $(242.4 \mathrm{~m})$ ) near Pakhal Lake.

Remarks: Wing: 62mm

1442 Cyornis tickelliae tickelliae (Blyth, 1843) Tickell's Blue-Flycatcher

SA: Muscicapula tickelliae tickelliae (Blyth) Tickell's Blue Flycatcher. O: Muscicapa tickelliae tickelliae Blyth.

NHM.OU.HSOS.35u. [112] Specimen of unknown sex collected on 13.x.31 from Mannanur (c' 2,060ft (624.2m)).

NHM.OU.HSOS.35v. [114] Male collected on 13.x.31 from Mannanur (c' 2,100ft (636.4m)).

Remarks: Unknown sex: Wing: $73 \mathrm{~mm}$; skull soft; in heavy general moult on head, body, wings; Ali (1933a) feels that the "plumage is indistinguishable from the adult female". Male: Wing: $73 \mathrm{~mm}$; testes developed moulting on wings and all over the body; Ali (1933a) opines that it is a breeding male. collected on 14.x.31 from Mannanur (c' 2,100ft 
1445 Eumyais thalassina thalassina(Swainson, 1838) Verditer Flycatcher

O: Muscicapa thalassina thalassina Swainson, 1838. NHM.OU.HSOS.35w. [420] Male collected on 3.xii.31 from Narsampet (c' 800ft (242.4)).

Remarks: Wing: $84 \mathrm{~mm}$. Testes undeveloped. Fresh plumage.

1448 Culicicapa ceylonensis calochrysea (Oberholser, 1923) Grey-headed Flycatcher

SA: Culicicapa ceylonensis pallidor Ticehurst. O Northern Grey-headed Flycatcher.

NHM.OU.HSOS.35x. [434] Specimen of unknown sex collected on 7.xii.31 from Pakhal Lake (c' 800ft (242.4m)) from Narsampet.

Remarks: Wing: $67 \mathrm{~mm}$

1452 Rhipidura aureola compressirostris (Blyth, 1849)

White-browed Fantail-Flycatcher

SA: Leucocerca aureola compressirostris Blyth Whitebrowed Fantail Flycatcher. O: Southern White-browed Fantail Flycatcher.

NHM.OU.HSOS.35y. [470] Male collected on 15.xii.31 from Asifabad (c' 1,200ft (363.6m)).

Remarks: Wing: $86 \mathrm{~mm}$. Testes undeveloped.

1458 Rhipidura albicollis albogularis (Lesson, 1832)

White-throated Fantail-Flycatcher

SA: Leucucerca pectoralis pectoralis Jerdon Whitespotted Fantail Flycatcher.

NHM.OU.HSOS.35z. [119] Female collected on 13.x.31 from Mannanur (c' 2,060ft (624.2m)).

Remarks: Wing: $74 \mathrm{~mm}$. Ovaries undeveloped. In heavy moult on body, wings, neck, tail.

1460 Terpsiphone paradisi leucogaster (Swainson, 1838)

Asian Paradise-Flycatcher

SA: Tchitrea paradisi leucogaster (Swainson) Paradise Flycatcher. O: West Himalayan Paradise Flycatcher. NHM.OU.HSOS.35aa. [663] Male collected on 6.iv.32 from Utnoor (c' 1,250ft (378.8m)).

Remarks: Wing: $100 \mathrm{~mm}$. Fresh plumage. Ali (1933a) opined it to be a "winter visitor".

1465 Hypothymis azurea styani (Hartlaub, 1898) Black-naped Monarch-Flycatcher

O: Monarcha azurea styani (Hartlaub) Indian Blacknaped Monarch Flycatcher

NHM.OU.HSOS.35bb. [248] Male collected on 5.xi.31 from Borgampad (c' 200ft $(60.6 \mathrm{~m})$ ).

Remarks: Wing: $71.5 \mathrm{~mm}$. Testes undeveloped. Fresh plumage.

1498 Cisticola juncidis cursitans (Franklin, 1831) Streaked Fantail-Warbler

SA: Streaked fantail Warbler.

NHM.OU.HSOS.35cc. [444] Male collected on 8.xii.31

from Narsampet (c' 750ft (227.3m)).

Remarks: Wing: $51 \mathrm{~mm}$. Testes undeveloped. Very worn plumage. Ali (1933b) notes that the specimen has "extremely worn secondaries".

1503 Prinia hodgsonii hodgsonii Blyth, 1844 Franklin's Prinia

SA: Franklinia gracilis Franklin. Franklin's Wren Warbler. O: Ashy Grey Wren Warbler.

NHM.OU.HSOS.35dd. [72] Male collected on 9.x.31 from Mannanur (c' 2,060ft (624.2m)).

NHM.OU.HSOS.35ee. [745] Female collected on 24.iv.32 from Kannad (c'2,500ft (757.6m)).

Remarks: Male: Testes developed; Breeding. Female: Wing: $47 \mathrm{~mm}$; ovaries undeveloped; in heavy general moult on body, wings, tail.

1511 Prinia inornata Sykes, 1832 Plain Prinia
SA: Prinia inornata inornata Sykes Indian Wren Warbler O: Prinia subflava inornata Sykes Central Indian Plain Wren-warbler.

NHM.OU.HSOS.35ff. [45] Female collected on 7.x.3 from Mannanur (c' 2,060ft (624.2m))

Remarks: Wing: 47mm. Ovaries undeveloped.

1521 Prinia sylvatica sylvatica Jerdon, 1840

Jungle Prinia

SA: Jungle Wren Warbler. O: Peninsular Jungle Wrenwarbler.

NHM.OU.HSOS.35gg. [4] Male collected on 25.ix.31 near Mir Alam Tank (c' 1,800ft $(545.5 \mathrm{~m})$ ) in Hyderabad City Environs

NHM.OU.HSOS.35hh. [346] Immature male collected on 20.xi.31 from Paloncha (c' 300ft (90.9m)).

NHM.OU.HSOS.35ii. [372] Female collected on

25.xi.31 from Paloncha (c'300ft $(90.9 \mathrm{~m})$ ).

Remarks: Male: Wing: 62mm; testes developed, pulpy. Iris orange; tongue plumbeous; bill black, inside mouth black; feet and claws fleshy brown, horny. Immature male: Testes undeveloped; skull soft; very worn plumage in heavy general moult. Female: Wing: $55 \mathrm{~mm}$ ovaries undeveloped; in heavy general moult.

1538 Orthotomus sutorius sutorius(Pennant, 1769) Common Tailor Bird

O: Ceylon Tailor Bird or Sri Lankan Tailor Bird.

NHM.OU.HSOS.35ij. [311] Male collected on 14.xi.31 from Nelipaka (c' 200ft $(60.6 \mathrm{~m}))$.

Remarks: Testes developed, pulpy. In heavy genera moult. Ali (1933b) mentions that the specimen is in "heavy post-nuptial moult".

1550 Acrocephalus stentoreus brunnescen (Jerdon, 1839)

Indian Great Reed-Warble

SA: Indian Great Reed Warbler.

NHM.OU.HSOS.35kk. [333] Male collected on 17.xi.31 from Nelipaka (c' 162ft $(49.1 \mathrm{~m}))$

Remarks: Wing: 92mm. Testes undeveloped. Shot in Tamarisk bed in Godavari river.

1556 Acrocephalus dumetorum Blyth, 1849

Blyth's Reed-Warbler

SA: Blyth's Reed Warbler.

NHM.OU.HSOS.3511. [389] Immature male collected

on 30.xi.31 from Narsampet (c' 780ft $(236.4 \mathrm{~m})$ ).

Remarks: Wing: 63mm. Testes undeveloped. Skull

soft in center

1563 Hippolais caligata rama (Sykes, 1832)

Booted Warbler

SA: Hippolais rama rama(Sykes). Sykes' Tree Warbler O: Indian Booted Tree Warbler.

NHM.OU.HSOS.35mm. [264] Male collected on 8.xi.31 from Nelipaka (c'160ft $(48.5 \mathrm{~m})$ )

Remarks: Wing: 65mm. Testes undeveloped. Fresh plumage.

1570 Sylvia curruca althaea Hume, 1878

Hume's Lesser Whitethroat

SA: Sylvia curruca affinis Blyth Indian Lesse Whitethroat.

NHM.OU.HSOS.35nn. [466] Female collected on 15.xii.31 from Asifabad (c' 1,200ft (363.6m)).

Remarks: Wing: 62mm. Ovaries undeveloped.

1581 Phylloscopus griseolus Blyth, 1847

Olivaceous Leaf-Warbler

SA: Olivaceous Tree Warbler.

NHM.OU.HSOS.35oo. [283] Male collected on 10.xi.31 from Nelipaka (c' 400ft (121.2m)).

Remarks: Wing: 68mm. Testes undeveloped.

1606 Phylloscopus occipitalis occipitalis (Blyth, 1845)

Western Crowned Warbler

SA: Large Crowned Willow Warbler. O: Large Crowned
Leaf Warbler

NHM.OU.HSOS.35pp. [436] Female collected on 7.xii.31 from Pakhal Lake (c' $800 \mathrm{ft}(242.4 \mathrm{~m})$ ) near Narsampet.

Remarks: Wing: 60mm. Ovaries undeveloped.

1644 Luscinia svecica (Linn., 1758)

Bluethroat

SA: Cyanosylvia svecica??. O: Erithacus svecicus (Linn.).

NHM.OU.HSOS.35qq. [191] Male collected on 30.x.31 from Borgampad (c'160ft $(48.5 \mathrm{~m})$ ).

NHM.OU.HSOS.35rr. [402] Immature female collected on 1.xii.31 from Narsampet (c' 780ft (236.4m)).

Remarks: Male: Wing: $77 \mathrm{~mm}$. Immature female: Wing $75 \mathrm{~mm}$; ovaries undeveloped; skull soft.

1661 Copsychus saularis saularis (Linn., 1758)

Oriental Magpie-Robin

SA: Magpie-robin. O: Indian Magpie-Robin.

NHM.OU.HSOS.35ss. [314] Male collected on 14.xii.31 from Nelipaka (c'162ft $(49.1 \mathrm{~m})$ ).

NHM OU.HSOS 35tt. [385] Female collected on 27.xi.31 from Paloncha (c' 300ft (90.9m)).

Remarks: Male: Wing: $99 \mathrm{~mm}$; testes undeveloped

fresh plumage. Female: Wing: $92 \mathrm{~mm}$; ovaries undeveloped; moulting retrices and on rump, otherwise fresh plumage.

1665 Copsychus malabaricus malabaricus (Scopoli, 1786)

White-rumped Shama

SA: Kittacincla malabarica malabarica (Scopoli) Shama O: Malabar Shama.

NHM.OU.HSOS.35uu. [340] Male collected on 19.xi.31 from Paloncha (c' 350ft (106.1m)).

Remarks: Wing: $91 \mathrm{~mm}$. Testes undeveloped. Mostly fresh plumage. Moulting on sides of neck. Ali (1933a) mentions "Specimen has a curiously short tail $-144 \mathrm{~mm}$ - for this race. On average female have tails ranging between $160-207 \mathrm{~mm}$ (12 specimens from Western Ghats) and male have between 160 -

200.5mm (9 specimens from Eastern Ghats)".

1672 Phoenicurus ochruros rufiventris (Vieillot, 1818)

Black Redstart

SA: Redstart. O: Eastern Black Redstart.

NHM.OU.HSOS.35vv. [398] Female collected on 1.xii.31 from Narsampet (c' $780 \mathrm{ft}(236.4 \mathrm{~m})$ ).

NHM.OU.HSOS.35ww. [43] Male collected on 6.x.31

from Mannanur (c' 2,060ft (624.2m)).

Remarks: Female: Wing: $86 \mathrm{~mm}$; ovaries undeveloped.

Male: Wing: $89 \mathrm{~mm}$; testes undeveloped.

1697 Saxicola torquata indica (Blyth, 1847)

Common Stonechat

SA: Indian Bush-chat. O: Stone or Collared Bush Chat. NHM.OU.HSOS.35xx. [121] Female collected on 13.x.31 from Mannanur (c' 2,060ft (624.2m)).

NHM.OU.HSOS.35yy. [384] Male collected on 27.xi.31 from Paloncha (c' 300ft $(90.9 \mathrm{~m})$ )

Remarks: Female: Wing: 68mm; ovaries undeveloped. Male: Wing: $70 \mathrm{~mm}$; testes undeveloped; moulting on back and rump.

1701 Saxicola caprata burmanica ( Baker, 1923) Pied Bushchat

SA: Burmese Stone-chat. O: Burmese Pied Bush Chat. NHM.OU.HSOS.35zz. [85] Male collected on 11.x.31 from Mannanur (c' 2,060ft (624.2m)).

NHM.OU.HSOS.35aaa. [196] Immature female collected on 30.x.31 from Borgampad (c' 160ft $(48.5 \mathrm{~m}))$.

Remarks: Male: Testes undeveloped; moulting on throat. Immature female: Wing $69 \mathrm{~mm}$; ovaries undeveloped; in fresh plumage; skull soft. 


1719 Saxicoloides fulicata intermedia (Whistler \&
Kinnear, 1932)
Indian Robin
SA: Robin. O: Deccan Black Robin.
NHM.OU.HSOS.35bbb. [288] Male collected on 11.xi.31
from Nelipaka (c' $160 \mathrm{ft}(48.5 \mathrm{~m})$ ).
NHM.OU.HSOS.35ccc. [633] Female collected on
2.iv.32 from Utnoor (c' $1,250 \mathrm{ft}(378.8 \mathrm{~m})$ ).
Remarks: Male: Wing: $74 \mathrm{~mm}$; testes undeveloped;
body plumage mostly fresh; moulting primaries; retrices
very worn. Female: Ovaries developed; breeding.

1734 Zoothera citrina cyanotus (Jardine \& Selby, 1828)

Orange-headed Thrush

SA: Geokichla citrina cyanotus (Jardine \& Selby)Whitecheeked Ground Thrush. O: White-throated Ground Thrush.

NHM.OU.HSOS.35ddd. [342] Male collected on 29.xi.31 from Paloncha (c' 300ft $(90.9 \mathrm{~m})$ ).

Remarks: Wing: $112 \mathrm{~mm}$. Testes undeveloped. Fresh plumage.

\section{Family: Paridae}

1795 Parus major mahrattarum (Hartert, 1905)

Great Tit

SA: Southern Grey Tit. O: Ceylon Grey Tit

NHM.OU.HSOS.36. [61] Immature male collected on 8.x.31 from Mannanur (c' 2,060ft (624.2m)).

NHM.OU.HSOS.36a. [272] Male collected on 9.xi.31 from Nelipaka (c' 200ft $(60.6 \mathrm{~m}))$.

NHM.OU.HSOS.36b. [329] Immature female collected on 16.xi.31 from Nelipaka (c' 200ft $(60.6 \mathrm{~m})$ ).

NHM.OU.HSOS.36c. [336] Immature male collected on 17.xi.31 from Nelipaka (c' $200 \mathrm{ft}(60.6 \mathrm{~m})$ ).

NHM.OU.HSOS.36d. [347] Female collected on 20.xi.31 from Paloncha (c' 300ft (90.9m)).

NHM.OU.HSOS.36e. [348] Immature specimen of unknown sex collected on 20.xi.31 from Paloncha (c' 300ft (90.9m)).

Remarks: Immature male -1: Wing: $64 \mathrm{~mm}$; testes undeveloped; skull very soft; in heavy moult. Male: Wing: 62mm; testes undeveloped; skull normally ossified; general moult. Immature female: Wing: $61 \mathrm{~mm}$; skull soft; moulting retrices, and on back and flanks. Immature male - 2: Wing: $65 \mathrm{~mm}$; testes undeveloped; in general moult; skull soft in center. Female: Wing: $63 \mathrm{~mm}$; ovaries undeveloped; completing general moult. Unknown sex: Wing: $65 \mathrm{~mm}$. ?Skull soft. In heavy general moult.

1810 Parus xanthogenys aplonotus (Blyth, 1847) Black-lored Tit

SA: Maclolophus xanthogenys aplonotus (Blyth) Yellow-cheeked Tit. O: Indian Yellow-cheeked Tit.

NHM.OU.HSOS.36f. [70] Male collected on 9.x.31 from Mannanur (c' 2,060ft (624.2m)).

NHM.OU.HSOS.36g. [158] Male collected on 17.x.31 from Farahabad (c' 2,800ft $(848.5 \mathrm{~m})$ ).

NHM.OU.HSOS.36h. [159] Female (doubtful) collected on 17.x.31 from Farahabad (c' 2,800ft $(848.5 \mathrm{~m})$ ).

NHM.OU.HSOS.36i. [170] Male collected on 18.x.31 from Farahabad (c' 2,800ft $(848.5 \mathrm{~m})$ ).

Remarks: Male -1: Testes undeveloped. Male -2: None. Female: Wing: $66 \mathrm{~mm}$; skull soft; in heavy general moult; Ali (1933a) writes that "the black band from the chin to the vent is replaced by dull olive green; measurements and plumages suggest that (it is an) adult female. Male -3: Wing: 70mm; testes undeveloped; skull soft; in heavy general moult; Ali (1933a) opines "Blackbreasted suggesting past juvenile moult.

Family: Sittidae

1830 Sitta castanea castanea (Lesson, 1830)

Chestnut-bellied Nuthatch

O: Peninsular Chestnut-bellied Nuthatch.

NHM.OU.HSOS.37. [602] Male collected on 23.iii.32 from Kaulas (c' 1,450ft (439.4m)).

Remarks: Wing: 75mm. Testes undeveloped. Moulting

upper tail coverts, otherwise fresh plumage.
1838 Sitta frontalis frontalis (Swainson, 1820) Velvet-fronted Nuthatch

No nomen change.

NHM.OU.HSOS.37a. [152] Male collected on 17.x.31

from Farahabad (c' 2,800ft $(848.5 \mathrm{~m})$ ).

Remarks: Wing: $75 \mathrm{~mm}$. Testes undeveloped. Moulting

on throat and body.

Family: Motacillidae

1854 Anthus trivialis trivialis (Linnaeus, 1758)

Eurasian Tree Pipit

SA: Tree Pipit. O: European Tree Pipit.

NHM.OU.HSOS.38. [505] Female collected on 3.iii.32 from Kandahar (c' 1,400ft (424.2m)).

Remarks: Wing: $85 \mathrm{~mm}$. Ovaries undeveloped. Moulting

on flanks, otherwise fresh plumage.

1884 Motacilla cinerea (Tunstall, 1771)

Grey Wagtail

SA: Motacilla cinerea caspica Gmelin Eastern Grey Wagtail.

NHM.OU.HSOS.38a. [135] Female collected on 16.x.31 from Farahabad (c' 2,688ft (814.5m)).

Remarks: Ovaries undeveloped.

1885 Motacilla alba dukhunensis (Sykes, 1832) White Wagtail

SA: Indian White Wagtail.

NHM.OU.HSOS.38b. [322] Immature female collected

on 16.xi.31 from Nelipaka (c' 162ft (49.1m)).

Remarks: Wing: $87 \mathrm{~mm}$. Ovaries undeveloped. Moulting

on upper back. Skull soft in center.

1891 Motacilla maderaspatensis (Gmelin, 1789) Large Pied Wagtail

No nomen change

NHM.OU.HSOS.38c. [30] Female collected on $5 \times 31$ from Mannanur (c' 2,100ft (636.4m)).

Remarks: Wing: 96mm. Ovaries undeveloped.

Family: Dicaeidae

1892 Dicaeum agile agile (Tickell, 1833)

Thick-billed Flowerpecker

SA: Pipirisoma agile agile Tickell. O: Indian Thick-billed Flowerpecker

NHM.OU.HSOS.39. [298] Female collected on 13.xi.31 from Nelipaka (c' 200ft $(60.6 \mathrm{~m}))$

Remarks: Ovaries undeveloped.

Family: Nectariniidae

1908 Nectarinia zeylonica zeylonica (Linnaeus 1766)

Purple-rumped Sunbird

SA: Cinnyris zeylonica (Linn.) O: Ceylon Purple-rumped Sunbird.

NHM.OU.HSOS.40. [38] Male collected on 6.x.31 from Mannanur

(c' 2,060ft $(624.2 \mathrm{~m})$ ).

NHM.OU.HSOS.40a. [305] Male collected on 14.xi.31 from Nelipaka (c' 200ft $(60.6 \mathrm{~m})$ ).

Remarks: Male -1: Testes developed. Male -2: Wing $56 \mathrm{~mm}$; testes developed.

1917 Nectarinia asiatica asiatica (Latham, 1790)

Purple Sunbird

SA: Cinnyris asiatica asiatica (Latham)

NHM.OU.HSOS.40b. [424] Male collected on 5.xii.31

from Narsampet (c' 780ft (236.4m)).

Remarks: Testes undeveloped

Family: Zosteropidae

1934 Zosterops palpebrosa salimali (Whistler, 1933) Oriental White-eye

SA: White-eye. O: Andhra White Eye

NHM.OU.HSOS.41. [73] Female collected on 9.x.31

from Mannanur (c' 2,060ft (624.2m)).

Remarks: Ovaries undeveloped.
Family: Ploceidae

1938 Passer domesticus indicus (Jardine \& Selby,

1835)

House Sparrow

SA: Indian House Sparrow

NHM.OU.HSOS.42. [21] Male collected on 30.ix.31 in Hyderabad City Environs (c' 1,800ft $(545.5 \mathrm{~m})$ ).

NHM.OU.HSOS.42a. [302] Female collected on 13.xi.31 from Nelipaka (c' 160ft (48.5m)).

Remarks: Male: Testes developed. Female: Wing: $69 \mathrm{~mm}$; ovaries undeveloped.

1949 Petronia xanthocollis xanthocollis (Burton, 1838)

Yellow-throated Sparrow

SA: Gymnorohis xanthocollis xanthocollis (Burton). O: Indian Yellow-throated Sparrow.

NHM.OU.HSOS.42b. [106] Male collected on 12.x.31 from Mannanur (c' 2,060ft (624.2m)).

Remarks: Wing: $86.5 \mathrm{~mm}$. Testes undeveloped.

1957 Ploceus philippinus philippinus (Linnaeus,

1766)

Baya Weaver

No nomen change.

NHM.OU.HSOS.42c. [19] Female collected on 27.ix.31 near Umda Sagar (c' 1800ft $(545.5 \mathrm{~m}))$ in Hyderabad City Environs.

NHM.OU.HSOS.42d. [437] Male collected on 8.xii.31 near Narsampet (c'750ft $(227.3 \mathrm{~m})$ ).

Remarks: Female: Wing: 68mm; ovaries undeveloped iris brown; bill yellowish, flesh brown on culmen; feet and claws brownish. Male: Wing: $73 \mathrm{~mm}$; testes undeveloped; in heavy general moult from breeding plumage on body, wings and tail.

1964 Amandava amandava amandava (Linnaeus,

1758)

Red Munia

O: Estrilda amandava amandava (Linnaeus.).

NHM.OU.HSOS.42e. [446] Specimen of unknown sex collected on 8.xii.31 from Narsampet (c' $750 \mathrm{ft}$ $(227.3 \mathrm{~m}))$.

NHM.OU.HSOS.42f. [673] Male collected on 8.iv.32 from Utnoor (c' 1,250ft (378.8m)).

Remarks: Unknown sex: Wing: 46.5mm. Male: Wing: $47 \mathrm{~mm}$; testes undeveloped; body and retrices moulting.

1966 Lonchura malabarica malabarica (Linnaeus, 1758)

White-throated Munia

SA: Uroloncha malabarica (Linnaeus).

NHM.OU.HSOS.42g. [15] Female collected on 27.ix.31 near Umda Sagar (c'1800ft $(545.5 \mathrm{~m})$ ) in Hyderabad City Environs.

Remarks: Wing: 54mm. Ovaries developed. Breeding. Iris dark brown. Bill slaty blue. Feet and claws purplish pink.

1974 Lonchura punctulata punctulata (Linnaeus, 1758) Spotted Munia

SA: Uroloncha punctulata lineoventer (Hodgson).

NHM.OU.HSOS.42h. [235] Female collected on 3.xi.31

from Borgampad (c' 200ft $(60.6 \mathrm{~m})$ ).

NHM.OU.HSOS.42i. [295] Immature specimen of unknown sex collected on 12.xi.31 from Nelipaka (c' 400ft).

Remarks: Female: Wing: 55mm; ovaries undeveloped; body moult; tail and wings worn. Unknown sex: Wing: $55 \mathrm{~mm}$; in heavy body moult; wings and tail fresh; skull soft.

Family: Fringillidae

2011 Carpodacus erythrinus roseatus (Blyth, 1842)

Common Rosefinch

SA: Common Indian Rosefinch. O: Indian Rosefinch. NHM.OU.HSOS.43. [441] Female collected on 8.xii.31 from Narsampet (c' 750ft (227.3m)). Remarks: None. 